\title{
BMJ Open Does neutrophil-to-lymphocyte ratio predict 1-year mortality in patients with primary biliary cholangitis? Results from a retrospective study with validation cohort
}

\author{
Lin Lin, ${ }^{1,2}$ Meiyu Piao, ${ }^{1,2}$ Xihui Jiang, ${ }^{1,2}$ Houning Lv, ${ }^{1,2}$ Ningning Zhao, ${ }^{1,2}$ \\ Fang Yang, ${ }^{1,2}$ Chao Sun ${ }^{1,2}$
}

To cite: Lin L, Piao M, Jiang X, et al. Does neutrophil-tolymphocyte ratio predict 1-year mortality in patients with primary biliary cholangitis? Results from a retrospective study with validation cohort. BMJ Open 2017;7:e015304. doi:10.1136/ bmjopen-2016-015304

- Prepublication history and additional material are available. To view these files please visit the journal online (http://dx.doi. org/10.1136/bmjopen-2016015304).

$\mathrm{LL}$ and MP contributed equally.

Received 28 November 2016 Revised 12 April 2017 Accepted 31 May 2017

\section{CrossMark}

${ }^{1}$ Department of Gastroenterology and Hepatology, Tianjin Medical University General Hospital, Tianjin, China

${ }^{2}$ Tianjin Institute of Digestive Disease, Tianjin Medical University General Hospital, Tianjin, China

Correspondence to Dr Chao Sun; chaosun@tmu. edu.cn

\section{ABSTRACT}

Objectives Neutrophil-to-lymphocyte ratio (NLR) has been used to predict prognosis in various liver diseases, but its role in primary biliary cholangitis (PBC) is not clarified. We aimed to investigate the prognostic usefulness of NLR for 1-year mortality in PBC.

Methods The study recruited a retrospective cohort with 88 patients with $\mathrm{PBC}$ and a prospective validation cohort with 63 participants who were followed-up for 1 year. NLR and other laboratory measurements were analysed by multivariate regression model for identifying independent factors for early mortality. The cut-off threshold of NLR was determined by calculating the area under the receiver operating characteristics curve (AUROC) and used in a subsequent Kaplan-Meier survival analysis.

Results Univariate and multivariate analyses showed that Mayo Risk Score (MRS), serum creatinine and NLR were independent indicators for mortality. NLR yielded significantly higher AUROC (0.86) than those of plateletto-lymphocyte ratio $(0.58, p=0.03)$, but comparable with MRS $(0.87, p=0.88)$. Spearman's correlation analysis represented a positive correlation between escalating NLR and aggravating Child-Pugh grade $(r=0.44, p<0.001)$. Patients with NLR $<2.18$ exhibited higher survival (with $100 \%$ sensitivity and $67.1 \%$ specificity) within 1 year follow-up duration, and NLR $\geq 2.18$ was indicative of higher mortality (log-rank test, $p<0.001)$. In addition, these results were internally confirmed by a validation cohort.

Conclusion NLR is closely related to short-term mortality in patients with PBC.

\section{INTRODUCTION}

Primary biliary cholangitis (PBC) is a chronic cholestatic liver disease characterised by immune-mediated destruction of intrahepatic bile duct, leading to inflammation, fibrosis and potentially cirrhosis. The diagnosis of PBC is based on a congruous set of elevated cholestatic indices and autoimmune serological hallmarks. Antimitochondrial antibody (AMA) is present in approximately $95 \%$ of patients with $\mathrm{PBC}$, whereas its prevalence in
Strengths and limitations of this study

- This is the first study in order to investigating the relationship between the neutrophil-to-lymphocyte ratio (NLR) value and 1-year mortality in primary biliary cholangitis (PBC) population. In addition, our findings were confirmed with an internal validation cohort.

- Our results demonstrated that elevated baseline NLR of hospitalised patients with PBC was associated with poor survival.

- Our results were derived from relatively small study population with short follow-up. The subsets of neutrophil and lymphocytes were not routinely measured in our department. Therefore, basically underlying mechanisms require further investigation.

healthy adults is extremely low, ranging from $0.16 \%$ to $1 \%{ }^{1}$ PBC predominantly involves females, who are in the fifth or sixth decade of their lives. Fatigue and pruritus manifest most prevalently in patients with PBC. The only accepted medical treatment is ursodeoxycholic acid (UDCA), which has been suggested to extend liver transplantation-free survival. However, up to $40 \%$ of patients with PBC do not biochemically respond to UDCA therapy. ${ }^{2}$ Furthermore, the development of the disease and the prognosis vary remarkably owing to individual discrimination. Collectively, it is pivotal to reliably predict outcomes in patients with PBC, which is of paramount importance in clinical strategies.

Neutrophil-to-lymphocyte ratio (NLR) refers to the imbalance of two distinct immune pathways. ${ }^{3}$ The neutrophil count (NC) reveals ongoing inflammation, whereas the lymphocyte count (LC) reflects the regulatory immune pathway as well as malnutrition. ${ }^{4}$ Accumulating evidence has suggested 
that increased NLR is associated with poor outcomes in various malignancies, cardiac disease and acute systemic inflammation. ${ }^{56}$ More recently, researchers have explored the feasibility of elevated NLR in predicting adverse outcomes, worsen histological grade or poor therapeutic response in several liver diseases. To the best of our knowledge, there were no studies in the literature regarding the usefulness of NLR for evaluating shortterm mortality in patients with PBC. Thus, we herein conducted a preliminary study in order to investigating the relationship between NLR and 1-year mortality in our PBC population.

\section{METHODS}

\section{Study subjects}

We recruited 151 patients with $\mathrm{PBC}$ who were admitted and followed-up at Department of Gastroenterology and Hepatology, Tianjin Medical University General Hospital. The study was approved by local ethic committee and in accordance with the Declaration of Helsinki. All patients provided written informed consent.

All enrolled participants were divided into two cohorts. A retrospective cohort comprised 88 patients who were hospitalised from June 2009 to January 2014. The validation cohort consisted of 63 patients who were prospectively recruited from February 2014 to February 2016. The diagnosis of PBC was based on two of the following three criteria: (1) increased alkaline phosphatase (ALP) or gamma glutamyl transferase (GGT) as cholestatic evidence over 6 months, (2) positive AMA according to the serum test and (3) hepatic histology compatible with PBC, typically non-suppurative cholangitis and interlobular bile duct injury. The exclusion criteria were: age $<18$ years; coinfection with HIV or hepatitis B/C virus; presence or history of hepatocellular carcinoma); the occurrence of other malignancy during study period; active infection or uncontrolled diabetes, coronary artery symptoms, heart failure and other diseases that might affect the NLR value on index examination and patients who accepted liver transplantation within study duration.

\section{Clinical information and laboratory examinations}

Baseline clinical data and results of laboratory tests were collected from our electronic medical records for patients in both the retrospective and validation cohorts. Only practitioners in our department were accessible to these information. Laboratory examinations were retrieved as following: white blood cell (WBC), NC, LC, platelet, alanine aminotransferase (ALT), aspartate aminotransferase (AST), ALP, GGT, total bilirubin (TBIL), direct bilirubin (DBIL), haemoglobin, albumin, serum creatinine (Scr) and international normalised ratio (INR). Other measurements included AMA and antinuclear antibody (ANA). Laboratory tests with missing data were not included for final analysis.

\section{Prognostic indices and models}

The Mayo Risk Score (MRS) was calculated as follows ${ }^{7}$ : $\mathrm{R}=0.039 \times$ age $($ years $)+0.871 \times \ln$ (bilirubin $(\mathrm{mg} /$ dL) $)+1.02 \times \ln$ (prothrombin time-INR) $+0.859 \times$ ascites $($ no $=0$, yes $=1)+6.843-2.53 \times \ln ($ albumin $(\mathrm{g} / \mathrm{dL}))$

The NLR and platelet-to-lymphocyte ratio (PLR) were measured as follows ${ }^{8}$ :

$$
\begin{aligned}
& \mathrm{NLR}=\mathrm{NC}\left(10^{9} / \mathrm{L}\right) / \mathrm{LC}\left(10^{9} / \mathrm{L}\right) \\
& \mathrm{PLR}=\text { platelet }\left(10^{9} / \mathrm{L}\right) / \mathrm{LC}\left(10^{9} / \mathrm{L}\right)
\end{aligned}
$$

\section{Statistical analysis}

All statistical analyses were carried out using MedCalc V.15.2.2 (MedCalc Software, Mariakerke, Belgium) and GraphPad Prism V.6.01 (Graphpad Software, La Jolla, California, USA) software. Data were demonstrated as mean \pm SD or simple number as appropriate. In univariate analysis between binary groups, an independent Student's t-test was performed for groups showing a normal distribution, and the Mann-Whitney test for groups without normal distribution. Categorical data were compared by $\mathrm{X}^{2}$ test or Fisher's exact test as appropriate. Multiple comparisons were performed by using Kruskal-Wallis test. Correlations were evaluated by the Spearman's correlation coefficient. Binary logistic regression with stepwise forward method was used for multivariate analysis as described elsewhere. ${ }^{9}$ The prognostic values for NLR, MRS, Scr and PLR were retrieved by using the area under the receiver operating characteristics curve (AUROC) with the best sensitivity and specificity in predicting 1-year mortality in patients with PBC. AUROC was addressed with its 95\% CI. The optimal cut-off for each parameter was determined when the Youden Index achieved highest value. AUROCs were compared in terms of the modality by DeLong et al. ${ }^{10} \mathrm{We}$ conducted the Kaplan-Meier method to estimate survival time and differences were compared using log-rank test. All $\mathrm{p}$ values were two-sided, and we considered a $\mathrm{p}$ value $<0.05$ as statistically significant.

\section{RESULTS}

The baseline characteristics and laboratory measurements of study population were shown in table 1 . In total, 11 of 151 patients $(7.3 \%)$ died during the 1-year follow-up period. Deaths were because of sepsis/multiorgan dysfunction in three and two cases, variceal bleeding in two and one cases and hepatorenal syndrome in one and two patients from retrospective and validation cohorts, respectively. Univariate analysis indicated comparable baseline levels of ALT, AST, ALP, GGT, haemoglobin, platelet, LC and PLR between the survival and death groups in the whole patients with PBC. However, age, TBIL, DBIL, albumin, NC, Scr, INR, NLR and MRS were significantly different in the two groups ( $\mathrm{p} \leq 0.05$ for all) (table 2). After adjusting for the effects of confounding factors by performing multivariate analysis, three variables including MRS (OR 2.34; 95\% CI 1.10 to $4.97 ; \mathrm{p}=0.028$ ), NLR (OR $1.50 ; 95 \%$ CI 1.00 to 2.23; $\mathrm{p}=0.039$ ) and Scr (OR 1.04; 95\% CI 1.00 to $1.07 ; \mathrm{p}=0.046$ ) remained significant prognostic factors for survival. 
Table 1 Demographic characteristics and laboratory measurements of enrolled patients

\begin{tabular}{|c|c|c|}
\hline Variable & $\begin{array}{l}\text { Retrospective } \\
\text { cohort }(n=88)\end{array}$ & $\begin{array}{l}\text { Validation cohort } \\
(n=63)\end{array}$ \\
\hline Age (years) & $60.7 \pm 10.9$ & $59.8 \pm 12.3$ \\
\hline $\begin{array}{l}\text { Gender (male/ } \\
\text { female) }\end{array}$ & $13 / 75$ & $6 / 57$ \\
\hline $\begin{array}{l}\text { Outcome (survival/ } \\
\text { death) }\end{array}$ & $82 / 6$ & $58 / 5$ \\
\hline ALT (U/L) & $117.7 \pm 140.9$ & $125.2 \pm 236.9$ \\
\hline AST (U/L) & $103.5 \pm 80.9$ & $116.4 \pm 205.0$ \\
\hline ALP (U/L) & $365.8 \pm 255.4$ & $281.0 \pm 162.6$ \\
\hline GGT (U/L) & $436.9 \pm 360.1$ & $335.9 \pm 275.6$ \\
\hline TBIL ( $\mu \mathrm{mol} / \mathrm{L})$ & $36.5 \pm 35.5$ & $36.7 \pm 40.8$ \\
\hline DBIL $(\mu \mathrm{mol} / \mathrm{L})$ & $20.8 \pm 26.3$ & $22.9 \pm 34.1$ \\
\hline Haemoglobin (g/L) & $108.1 \pm 23.0$ & $106.9 \pm 26.8$ \\
\hline Albumin (g/L) & $35.7 \pm 5.9$ & $36.7 \pm 6.5$ \\
\hline WBC $\left(10^{9} / \mathrm{L}\right)$ & $5.1 \pm 2.1$ & $5.1 \pm 2.2$ \\
\hline Platelet $\left(10^{9} / \mathrm{L}\right)$ & $154.6 \pm 75.1$ & $158.7 \pm 71.2$ \\
\hline $\mathrm{NC}\left(10^{9} / \mathrm{L}\right)$ & $2.8 \pm 1.7$ & $2.9 \pm 1.8$ \\
\hline LC $\left(10^{9} / \mathrm{L}\right)$ & $1.5 \pm 0.9$ & $1.5 \pm 0.8$ \\
\hline Scr & $61.2 \pm 23.7$ & $59.8 \pm 16.9$ \\
\hline INR & $1.09 \pm 0.19$ & $1.09 \pm 0.24$ \\
\hline NLR & $2.29 \pm 1.73$ & $2.32 \pm 1.91$ \\
\hline MRS & $6.70 \pm 1.42$ & $6.56 \pm 1.60$ \\
\hline PLR & $116.7 \pm 57.8$ & $116.5 \pm 56.0$ \\
\hline
\end{tabular}

Values were expressed as mean $\pm \mathrm{SD}$ or $\mathrm{n}$.

ALP, alkaline phosphatase; ALT, alanine aminotransferase; AST, aspartate aminotransferase; DBIL, direct bilirubin; GGT, gamma glutamyl transferase; INR, international normalised ratio; LC, lymphocyte count; MRS, Mayo Risk Score; NC, neutrophil count; NLR, neutrophil-to-lymphocyte ratio; PLR, platelet-tolymphocyte ratio; Scr, serum creatinine; TBIL, total bilirubin; WBC, white blood cell.

Next, we analysed the predictive value of NLR, MRS, Scr and PLR by applying ROC. As shown in table 3 and figure 1, the AUROC of PLR was only $0.58(\mathrm{p}=0.51)$, whereas those of NLR, MRS and Scr were comparable $(0.86,0.87$ and 0.83 , respectively; $\mathrm{p}<0.01$ for all). The AUROC of NLR was significantly higher than that of PLR ( $p=0.03)$. In addition, ROC analysis revealed that the optimal cut-off value of NLR for the prediction of 1-year mortality was 2.18 with a sensitivity, specificity, positive predictive value, negative predictive value, likelihood ratio of $100 \%, 67.1 \%, 18.2 \%, 100 \%$ and $3.04 \%$, respectively. Furthermore, figure 2 illustrated that escalating values of NLR were associated with aggravating Child-Pugh stratification in patients with $\mathrm{PBC}(\mathrm{r}=0.44$, $\mathrm{p}<0.001)$. Taken together, similar to MRS, NLR represented a potential index between the survival and death groups in the retrospective cohort.

Since the NLR was significantly higher in the death group than that in the survival group $(p=0.002)$, we examined the levels of WBC, NC and LC to determine the factors predominantly affecting NLR value (table 2 and see online supplementary figure 1 ). The baseline WBC and LC were similar in both groups $(5.02 \pm 2.05$ vs $5.88 \pm 2.72, \mathrm{p}=0.33 ; 1.57 \pm 0.87$ vs $1.16 \pm 0.70, \mathrm{p}=0.27)$. However, the death group exhibited a higher level of NC in comparison with that in the survival group $(4.99 \pm 2.41$ vs $2.68 \pm 1.55, \mathrm{p}=0.001)$. Collectively, these results showed that the elevated NLR in the death group was mainly owing to an increased NC in the present study.

The retrospective cohort was divided into two groups in terms of the NLR cut-off value: group 1 (NLR <2.18) and group 2 (NLR $\geq 2.18$ ). In group 1 , none of the 55 patients died, whereas in group 2, 6 of the 33 patients died $(18.2 \%)$ during 1-year observation period. A KaplanMeier survival analysis was shown in figure 3. Patients with baseline NLR $<2.18$ had a significantly longer survival time compared with those with NLR of at least 2.18 (log-rank test, $\mathrm{p}<0.001$ ).

Finally, to confirm the results based on the retrospective cohort, 63 patients with $\mathrm{PBC}$ were recruited as a prospective validation cohort. The AUROC of NLR for the validation cohort was $0.90(\mathrm{p}=0.005)$. The patients in group with a setting of NLR value $<2.18$ had a significantly longer survival time compared with those with NLR of at least 2.18 (log-rank test, $\mathrm{p}=0.011$ ) (see online supplementary figure 2 ).

\section{DISCUSSION}

In the current study, we aimed to investigate the relationship between NLR and 1-year mortality in patients with PBC. Our results demonstrated that elevated baseline NLR of hospitalised patients with PBC was associated with poor survival. Notably, NLR showed a comparable AUROC with MRS, which is a well identified and commonly used scoring model for predicting prognosis in various liver diseases. NLR was also superior to PLR and advantageous in simple and rapid access. The patients with NLR $<2.18$ exhibited lower mortality, whereas NLR $\geq 2.18$ was indicative of increased death risk. In addition, the predictive value of NLR was confirmed in a validation cohort.

NLR has emerged as an index of the systemic inflammatory circumstance and stress, and it has been validated to predict survival and outcomes in solid tumours, as well as critical illness, for instance, coronary artery diseases and acute pancreatitis. ${ }^{511-13}$ With respect to hepatic carcinoma, a meta-analysis has suggested that NLR potentially balances the functions of neutrophils and immunocytes. ${ }^{14}$ The bioactivity of tumour cells, including invasion, angiogenesis and metastasis is concisely regulated by signals arising from immune-associated cells, including neutrophils, lymphocytes and platelets. ${ }^{15-17}$ Neutrophils and platelets are always relevant to inflammation, whereas lymphocytes are indispensable for immunosurveillance and immunoediting. ${ }^{18}$ Therefore, NLR might serve as attractive prognostic value for patients with primary liver cancer. More recently, NLR was also considered to be 
Table 2 Univariate analysis for the relationship between variables and 1-year mortality in patients with primary biliary cholangitis

\begin{tabular}{|c|c|c|c|}
\hline Variable & Survival group $(n=140)$ & Death group $(n=11)$ & p Value \\
\hline Age (years) & $59.2 \pm 10.8$ & $74.0 \pm 10.9$ & $<0.001^{*}$ \\
\hline Gender (male/female) & $17 / 123$ & $2 / 9$ & $0.63 \dagger$ \\
\hline ALT (U/L) & $126.4 \pm 192.4$ & $50.6 \pm 29.2$ & $0.11 \ddagger$ \\
\hline AST (U/L) & $112.5 \pm 150.3$ & $62.7 \pm 41.7$ & $0.09 \ddagger$ \\
\hline ALP (U/L) & $330.1 \pm 230.9$ & $335.5 \pm 131.3$ & $0.94^{*}$ \\
\hline GGT (U/L) & $400.3 \pm 340.6$ & $324.8 \pm 135.2$ & $0.90 \ddagger$ \\
\hline TBIL $(\mu \mathrm{mol} / \mathrm{L})$ & $33.4 \pm 30.8$ & $76.4 \pm 78.9$ & $0.008^{\ddagger}$ \\
\hline DBIL $(\mu \mathrm{mol} / \mathrm{L})$ & $19.5 \pm 23.3$ & $51.5 \pm 67.9$ & $0.03^{\ddagger}$ \\
\hline Haemoglobin (g/L) & $108.4 \pm 24.3$ & $97.6 \pm 26.7$ & $0.16^{*}$ \\
\hline Albumin (g/L) & $36.7 \pm 6.0$ & $29.0 \pm 3.5$ & $<0.001^{*}$ \\
\hline WBC $\left(10^{9} / L\right)$ & $4.98 \pm 2.11$ & $6.21 \pm 2.27$ & $0.07^{*}$ \\
\hline Platelet $\left(10^{9} / \mathrm{L}\right)$ & $159.3 \pm 73.7$ & $118.3 \pm 57.7$ & $0.07^{*}$ \\
\hline $\mathrm{NC}\left(10^{9} / \mathrm{L}\right)$ & $2.68 \pm 1.59$ & $5.17 \pm 1.96$ & $<0.001^{*}$ \\
\hline $\operatorname{LC}\left(10^{9} / \mathrm{L}\right)$ & $1.57 \pm 0.85$ & $1.09 \pm 0.61$ & $0.07^{\star}$ \\
\hline Scr & $58.5 \pm 15.1$ & $87.6 \pm 51.2$ & $0.006 \ddagger$ \\
\hline INR & $1.07 \pm 0.17$ & $1.41 \pm 0.41$ & $<0.001 \ddagger$ \\
\hline NLR & $2.03 \pm 1.24$ & $5.75 \pm 3.59$ & $<0.001 \ddagger$ \\
\hline MRS & $6.45 \pm 1.35$ & $9.01 \pm 1.24$ & $<0.001^{*}$ \\
\hline PLR & $118.2 \pm 58.5$ & $121.5 \pm 63.6$ & $0.86^{*}$ \\
\hline
\end{tabular}

Values were expressed as mean $\pm \mathrm{SD}$ or $\mathrm{n}$.

*Student's t-test

†Fisher's exact test

$\ddagger$ Mann-Whitney test.

ALP, alkaline phosphatase; ALT, alanine aminotransferase; AST, aspartate aminotransferase; DBIL, direct bilirubin; GGT, gamma glutamyl transferase; INR, international normalised ratio; LC, lymphocyte count; MRS, Mayo Risk Score; NC, neutrophil count; NLR, neutrophil-tolymphocyte ratio; PLR, platelet-to-lymphocyte ratio; Scr, serum creatinine; TBIL, total bilirubin; WBC, white blood cell.

a reliable predictor for advanced histological grade or poor outcomes in a variety of hepatic entities. Liu et al concluded that elevated NLR served as a powerful marker for predicting short-term mortality in acute-on-chronic hepatitis B liver failure. ${ }^{6}$ Another study unveiled NLR as a simple and easily accessible parameter for evaluating therapeutic response in patients with chronic hepatitis C. ${ }^{19}$ In addition, a high NLR was regarded as an available indicator of worsen and advanced pathological status in non-alcoholic fatty liver disease. ${ }^{20}$ However, the utility and feasibility of NLR in autoimmune liver disease are still under investigation.
PBC is a chronic cholestatic disease characterised by typical appearance of non-suppurative destructive cholangitis. ${ }^{21}$ It is believed that persistent inflammation will result in progressive liver damage eventually advancing to fibrosis and cirrhosis. ${ }^{22}$ As a matter of fact, multiple immune cells, inflammatory cytokines as well as chemokines have been involved in the pathogenesis of PBC. ${ }^{23}$ Zimmermann et al found that patients with cholestatic diseases exhibited highest serum interleukin (IL)-8 concentrations, and intrahepatic IL-8 was positively associated with neutrophil accumulation in patients with PBC. ${ }^{24}$ Another study showed IL-33 and/or myeloperoxidase (a

Table 3 Diagnostic accuracy of different factors for the prediction of 1-year mortality in patients with primary biliary cholangitis

\begin{tabular}{lllllllll}
\hline Factor & AUROC $(\mathbf{9 5 \%}$ Cl) & p Value & Cut-off & Sensitivity (\%) & Specificity (\%) & PPV (\%) & NPV (\%) & LR \\
\hline NLR & $0.86(0.74$ to 0.97$)$ & 0.004 & 2.18 & 100.0 & 67.1 & 18.2 & 100 & 3.04 \\
MRS & $0.87(0.75$ to 0.99$)$ & 0.003 & 6.52 & 100.0 & 59.8 & 15.4 & 100 & 2.49 \\
Scr & $0.83(0.65$ to 0.99$)$ & 0.009 & 64.5 & 83.3 & 70.7 & 17.2 & 98.3 & 2.85 \\
PLR & $0.58(0.41$ to 0.75$)$ & 0.51 & 125.4 & 100.0 & 42.7 & 11.3 & 100.0 & 1.75
\end{tabular}

AUROC, area under the receiver operating characteristic curve; LR, likelihood ratio; MRS, Mayo Risk Score; NLR, neutrophil-to-lymphocyte ratio; NPV, negative predictive value; PLR, platelet-to-lymphocyte ratio; PPV, positive predictive value; Scr, serum creatinine. 


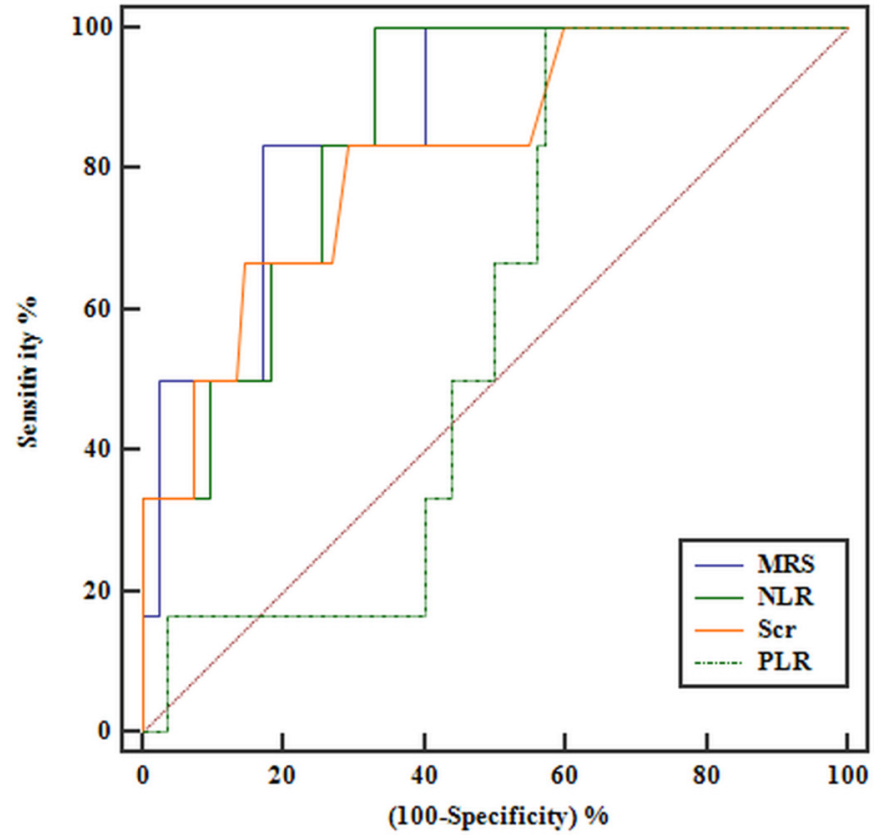

Figure 1 ROC analysis of prognostic variables in patients with primary biliary cholangitis in the retrospective cohort. MRS, Mayo Risk Score; NLR, neutrophil-to-lymphocyte ratio; PLR, platelet-to-lymphocyte ratio; ROC, receiver operating characteristics; Scr, serum creatinine.

marker for neutrophils) expressing cells increased in accordance with worsen histological severity, and IL-33 may influence the progress of PBC by recruiting neutrophils to the liver. ${ }^{25}$ Collectively, above mentioned evidence may partly state the results in current study, which representing elevated NC dominantly contribute to the higher NLR in patients from death subgroup. One issue that should be addressed is the controversial data regarding LC from literatures. Although others or us did not observe significantly changing of $\mathrm{LC}^{26}{ }^{26}$ some researchers did report dramatically declined LC in association with poor outcomes in various liver diseases. ${ }^{627}$ These contradictory

(a)

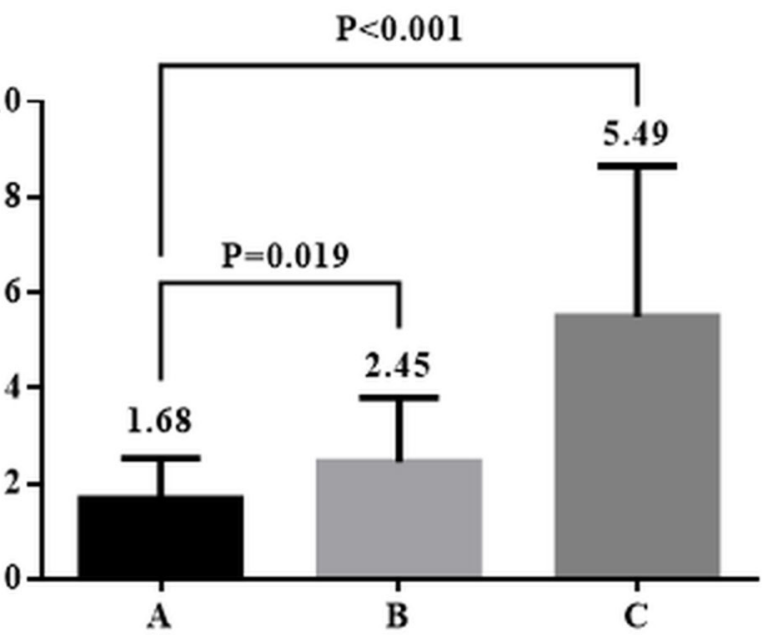

Child-Pugh grade

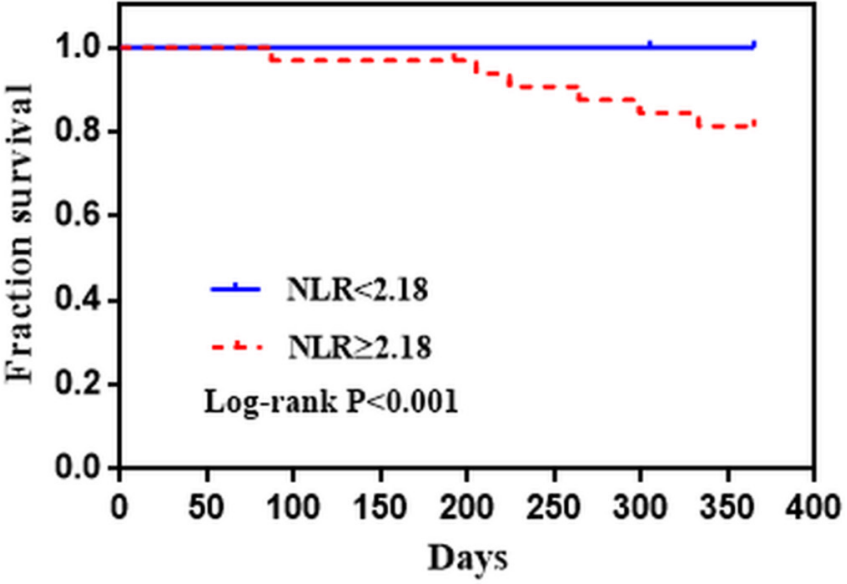

Figure 3 One-year Kaplan-Meier survival curve by NLR $(p<0.001$ for log-rank test) in the retrospective cohort. NLR, neutrophil to lymphocyte ratio.

results could be attributed to discrepant entities for analysis, and the absolute number of individual cell types might be vulnerable to circumstance, such as infection, medication, stress and various physiological conditions. ${ }^{28}$ Remarkably, NLR represents a ratio of two distinct complementary immune pathways, thus integrating the deleterious effects of NC. Taken together, the predictive efficacy of NLR is more objective than either parameter alone.

Until now, no universal predictive cut-off value of NLR has been proposed in a variety of diseases. A recent meta-analysis implied that a threshold of NLR greater than 5 was indicative of unfavourable impact in primary liver cancer, whereas another study showed that dichotomised cut-off value, that is 5 versus not 5 , will not undermine the predictive efficiency of NLR in colorectal cancer. ${ }^{14} 29$ In the present study, we obtained the cut-off value of NLR not less than 2.18 for determining poor survival outcome in patients with $\mathrm{PBC}$, with a promising sensitivity of

(b)

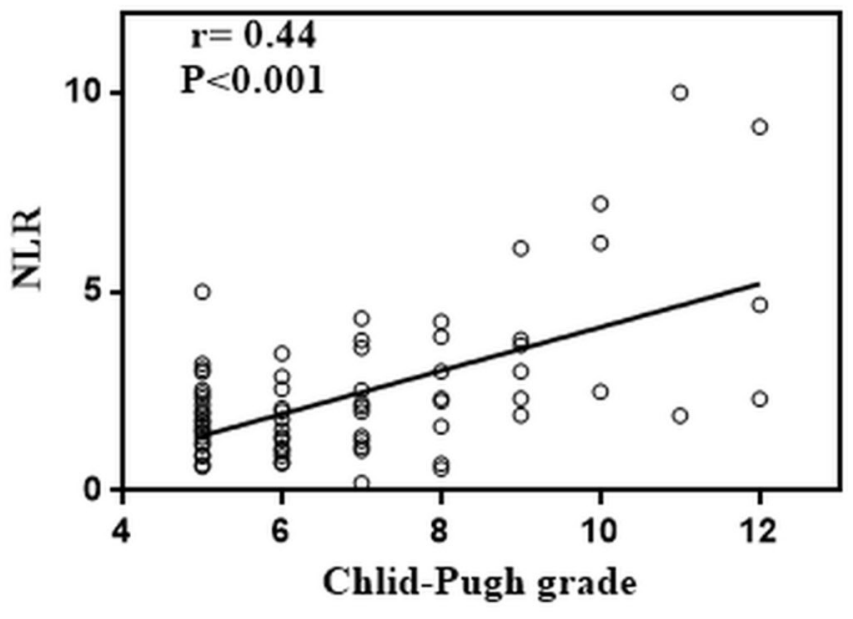

Figure 2 The relationship between NLR and Child-Pugh grade. NLR, neutrophil-to-lymphocyte ratio. 
$100 \%$. Taken these into consideration, the underlying mechanism of elevated NLR is much more complicated owing to its characteristics as a combined parameter of inflammation and host immune surveillance. ${ }^{30}$ Expanded neutrophils may give rise to supporting milieu, subsequently enhance the growth, proliferation, angiogenesis and invasion of cancer cells. It is well established that tumour-associated inflammatory microenvironment is related to alteration in peripheral blood cells, especially the presence of neutrophilia with a relative lymphocytopenia. ${ }^{31}$ Regarding PBC, several studies implied that 10 to 100 -fold increase in the frequency of autoreactively intrahepatic cluster of differentiation $4^{+}\left(\mathrm{CD}^{+}\right)$or $\mathrm{CD}^{+} \mathrm{T}$ cells compared with those in the peripheral blood. ${ }^{32}$ Collectively, these results could partly explain the higher cut-off point of NLR in malignancies, while the best threshold of NLR, whose value increased in terms of Child-Pugh class, was relatively lower in patients with PBC (2.18 in our cohort).

The current study still has some limitations. First, our results were derived from relatively small study population with short follow-up. However, a most recent study (107 patients enrolled) from the USA concluded that elevated NLR was associated with 90-day mortality in liver transplantation candidates. ${ }^{33}$ Moreover, the authors stated NLR was a readily and reliable marker over time. Second, this was a single-centre retrospective study with selection bias; therefore, accumulatively prospective data from multi-centre may confirm our findings and more precisely predict poor outcomes by using NLR. However, we already tried to eliminate any confounding factors against NLR value at initial evaluation, including overt infection, neoplasms as well as heart diseases. The homogeneity of the study population probably render the present results feasible. Moreover, a validation cohort was included for final analysis. Third, the subsets of neutrophil and lymphocytes were not routinely measured in our department. Therefore, basically underlying mechanisms require further investigation.

In conclusion, NLR, an affordable, widely available and reproducible index, is closely related to short-term mortality in patients with PBC. Further studies are warranted to externally cross-validate our findings in other population.

Contributors LL, MP and CS designed the study and drafted the manuscript. LL, $\mathrm{XJ}, \mathrm{HL}$ and NZ collected the clinical data. LL, FY, MP and CS analysed the data. $\mathrm{LL}$ and $\mathrm{CS}$ reviewed the data and revised the manuscript. FY, XJ and $\mathrm{HL}$ provided statistical advice. All authors approved the final version.

Competing interests None declared.

Patient consent Obtained.

Ethics approval Ethic Committee of TJMUGH.

Provenance and peer review Not commissioned; externally peer reviewed.

Data sharing statement № additional data are available.

Open Access This is an Open Access article distributed in accordance with the Creative Commons Attribution Non Commercial (CC BY-NC 4.0) license, which permits others to distribute, remix, adapt, build upon this work non-commercially, and license their derivative works on different terms, provided the original work is properly cited and the use is non-commercial. See: http://creativecommons.org/ licenses/by-nc/4.0/

(c) Article author(s) (or their employer(s) unless otherwise stated in the text of the article) 2017. All rights reserved. No commercial use is permitted unless otherwise expressly granted.

\section{REFERENCES}

1. Achenza MI, Meda F, Brunetta E, et al. Serum autoantibodies for the diagnosis and management of autoimmune liver diseases. Expert Rev Gastroenterol Hepatol 2012;6:717-29.

2. Carey EJ, Ali AH, Lindor KD. Primary biliary cirrhosis. Lancet 2015;386:1565-75.

3. Avanzas P, Quiles J, López de Sá E, et al. Neutrophil count and infarct size in patients with acute myocardial infarction. Int $J$ Cardiol 2004;97:155-6.

4. Kwon JH, Jang JW, Kim YW, et al. The usefulness of C-reactive protein and neutrophil-to-lymphocyte ratio for predicting the outcome in hospitalized patients with liver cirrhosis. BMC Gastroenterol 2015;15:146.

5. Muhmmed Suliman MA, Bahnacy Juma AA, Ali Almadhani AA, et al. Predictive value of neutrophil to lymphocyte ratio in outcomes of patients with acute coronary syndrome. Arch Med Res 2010;41:618-22.

6. Liu H, Zhang H, Wan G, et al. Neutrophil-lymphocyte ratio: a novel predictor for short-term prognosis in acute-on-chronic hepatitis $B$ liver failure. J Viral Hepat 2014;21:499-507.

7. Dickson ER, Grambsch PM, Fleming TR, et al. Prognosis in primary biliary cirrhosis: model for decision making. Hepatology 1989;10:1-7

8. Meng X, Wei G, Chang Q, et al. The platelet-to-lymphocyte ratio, superior to the neutrophil-to-lymphocyte ratio, correlates with hepatitis C virus infection. Int J Infect Dis 2016;45:72-7.

9. Cetinkaya E, Senol K, Saylam B, et al. Red cell distribution width to platelet ratio: new and promising prognostic marker in acute pancreatitis. World J Gastroenterol 2014;20:14450-4.

10. DeLong ER, DeLong DM, Clarke-Pearson DL. Comparing the areas under two or more correlated receiver operating characteristic curves: a nonparametric approach. Biometrics 1988;44:837-45.

11. Cao LL, Lu J, Lin JX, et al. A novel predictive model based on preoperative blood neutrophil-to-lymphocyte ratio for survival prognosis in patients with gastric neuroendocrine neoplasms. Oncotarget 2016;7:42045-42058.

12. Zahorec R. Ratio of neutrophil to lymphocyte counts-rapid and simple parameter of systemic inflammation and stress in critically ill. Bratis/ Lek Listy 2001;102:5-14.

13. Azab B, Jaglall N, Atallah JP, et al. Neutrophil-lymphocyte ratio as a predictor of adverse outcomes of acute pancreatitis. Pancreatology 2011;11:445-52.

14. Xue TC, Zhang L, Xie XY, et al. Prognostic significance of the neutrophil-to-lymphocyte ratio in primary liver cancer: a metaanalysis. PLoS One 2014;9:e96072.

15. Grosse-Steffen T, Giese T, Giese N, et al. Epithelial-to-mesenchymal transition in pancreatic ductal adenocarcinoma and pancreatic tumor cell lines: the role of neutrophils and neutrophil-derived elastase. Clin Dev Immunol 2012;2012:1-12.

16. Schwaller J, Schneider P, Mhawech-Fauceglia P, et al. Neutrophilderived APRIL concentrated in tumor lesions by proteoglycans correlates with human B-cell lymphoma aggressiveness. Blood 2007;109:331-8.

17. Schumacher D, Strilic B, Sivaraj KK, et al. Platelet-derived nucleotides promote tumor-cell transendothelial migration and metastasis via P2Y2 receptor. Cancer Cell 2013;24:130-7.

18. Dunn GP, Old LJ, Schreiber RD. The immunobiology of cancer immunosurveillance and immunoediting. Immunity 2004;21:137-48.

19. Kuo MT, Hu TH, Lu SN, et al. Neutrophil-to-lymphocyte ratio as a predictor of response to peginterferon plus ribavirin therapy for chronic hepatitis C. Dis Markers 2014;2014:1-6.

20. Alkhouri N, Morris-Stiff G, Campbell C, et al. Neutrophil to lymphocyte ratio: a new marker for predicting steatohepatitis and fibrosis in patients with nonalcoholic fatty liver disease. Liver Int 2012;32:297-302.

21. Kaplan MM, Gershwin ME. Primary biliary cirrhosis. N Engl J Med 2005;353:1261-73.

22. Hirschfield GM, Karlsen TH, Lindor KD, et al. Primary sclerosing cholangitis. Lancet 2013;382:1587-99.

23. Liaskou E, Hirschfield GM, Gershwin ME. Mechanisms of tissue injury in autoimmune liver diseases. Semin Immunopathol 2014;36:553-68. 
24. Zimmermann HW, Seidler S, Gassler N, et al. Interleukin-8 is activated in patients with chronic liver diseases and associated with hepatic macrophage accumulation in human liver fibrosis. PLoS One 2011;6:e21381.

25. Sun Y, Zhang JY, Lv S, et al. Interleukin-33 promotes disease progression in patients with primary biliary cirrhosis. Tohoku J Exp Med 2014;234:255-61.

26. Yilmaz B, Aydin H, Can G, et al. The relationship between fibrosis level and blood neutrophil to lymphocyte ratio in inactive hepatitis $B$ carriers. Eur J Gastroenterol Hepatol 2014;26:1-1328.

27. Biyik M, Ucar R, Solak Y, et al. Blood neutrophil-to-lymphocyte ratio independently predicts survival in patients with liver cirrhosis. Eur J Gastroenterol Hepatol 2013;25:435-41.

28. Bhat T, Teli S, Rijal J, et al. Neutrophil to lymphocyte ratio and cardiovascular diseases: a review. Expert Rev Cardiovasc Ther 2013;11:55-9.
29. Li MX, Liu XM, Zhang XF, et al. Prognostic role of neutrophil-tolymphocyte ratio in colorectal cancer: a systematic review and metaanalysis. Int J Cancer 2014;134:2403-13.

30. Oh BS, Jang JW, Kwon JH, et al. Prognostic value of C-reactive protein and neutrophil-to-lymphocyte ratio in patients with hepatocellular carcinoma. BMC Cancer 2013;13:78.

31. Guthrie GJ, Charles KA, Roxburgh CS, et al. The systemic inflammation-based neutrophil-lymphocyte ratio: experience in patients with Cancer. Crit Rev Oncol Hematol 2013;88:218-30.

32. Kita H, Matsumura S, He XS, Xs H, et al. Quantitative and functional analysis of PDC-E2-specific autoreactive cytotoxic T lymphocytes in primary biliary cirrhosis. J Clin Invest 2002;109:1231-40.

33. Kalra A, Wedd JP, Bambha KM, et al. Neutrophil-to-lymphocyte ratio correlates with proinflammatory neutrophils and predicts death in low model for end-stage liver disease patients with cirrhosis. Liver Transp/ 2017;23:155-65. 